\title{
Neuer Vorstand der KIBA - Sektion 7 im dbv und Ausbildungskommission der DGI
}

http://doi.org/10.1515/bd-2020-0020

Die Konferenz der informations- und bibliothekswissenschaftlichen Ausbildungsund Studiengänge (KIBA) hat auf ihrer Jahrestagung im November 2019 an der Hochschule Darmstadt Prof. Dr. Stefan Schmunk (Hochschule Darmstadt - HDA), Prof. Frauke Schade (HAW Hamburg) und Prof. Dr. Günther Neher (FH Potsdam) einstimmig als neuen Vorstand für die Amtszeit von 2019 bis 2022 gewählt. Den Vorsitz übernimmt Prof. Dr. Stefan Schmunk.

Nach zwei Amtsperioden hat sich die bisherige Vorsitzende Prof. Frauke Schade entschieden, nicht erneut für den Vorsitz zu kandidieren. Bedingt durch den Wechsel von der Hochschule für öffentliche Verwaltung zur Hochschule Hannover zum Wintersemester 2019/2020 und den damit verbundenen Veränderungen hat zudem Prof. Dr. Klaus Gantert sein bisheriges Vorstandsamt abgegeben. Die Mitglieder der KIBA dankten dem scheidenden Vorstand ausdrücklich für sein Engagement, insbesondere bei der Interessenvertretung der Hochschulen gegenüber Verbänden und der Berufsöffentlichkeit, beim Aufbau und der Pflege von Kontakten und Kooperationen und für die Thematisierung von Fragen der Nachwuchsförderung.

Die Herbsttagung wurde dazu genutzt, sich über Ziele, Aufgaben und Arbeitsschwerpunkte der KIBA auszutauschen. Arbeitsprogramm und Verantwortlichkeiten für die Jahre 2020 bis 2022 wird der neue Vorstand im Januar 2020 vorstellen.

Die KIBA ist die Interessenvertretung der bibliotheks- und informationswissenschaftlichen Hochschulen und Ausbildungseinrichtungen in Deutschland. Sie dient ihren Mitgliedern als Forum für den Austausch und erarbeitet Positionen $\mathrm{zu}$ ausbildungsrelevanten Themen der Hochschulen in der Fachöffentlichkeit. Die KIBA betreibt Lobbyarbeit gegenüber Berufsverbänden und Politik, Berufspraxis und anderen Einrichtungen außerhalb des Hochschulbereichs. Sie vertritt außerdem die Belange und Interessen ihrer Mitglieder bei internationalen Organisationen wie der International Federation of Library Associations and Institutions (IFLA). Die KIBA ist Sektion 7 des Deutschen Bibliotheksverbandes und Ausbildungskommission der Deutschen Gesellschaft für Information und Wissen (DGI). 